\title{
Analysis and Comparison of Routing Protocol of Wireless Sensor Network
}

\author{
https://doi.org/10.3991/ijoe.v12i10.6195 \\ Lei An ${ }^{1}$, Aihua $\mathrm{Li}^{1}$, Ling Pang ${ }^{2}$, Guangdong $\mathrm{He}^{1}$ \\ ${ }^{1}$ BaoDing University \\ ${ }^{2}$ HeBei Finance University
}

\begin{abstract}
There are many differences between the routing protocol design of a wireless sensor network and wireless ad-hoc network. Highly restricted resource and frequent node failure are two major challenges. Relevant technical research into wireless sensor network has become an issue of intense interest. In recent years, achievements in research on routing protocol of wireless sensor network have been summarized, analyzed and compared. Features of wireless sensor networks have been introduced and key factors of its routing protocol design have been introduced. According to the realization features of protocol, routing protocol of wireless sensor network has been divided into five categories, and each category of important protocols has been elaborated and analyzed. Finally, the features of these protocols are summarized and compared and the development trend of such research has been forecasted.
\end{abstract}

Index Terms-wireless sensor network, sensor node, sink node, routing protocol.

\section{INTRODUCTION}

With the continuous development of sensor technology and wireless communication technology, wireless sensor network has drawn great attention. A wireless sensor network is composed of a group of sensor nodes via a wireless connection. It configures a large number of micro intelligent sensor nodes in an ad hoc manner and collects and processes target information in the area covered by the network via collaboration. The wireless sensor network has a prospective wide application in military monitoring, environmental monitoring, earthquake and climate forecasting, rescue and relief and underground exploration, as well as in deep water and outer space [1].

Energy resource, computing power and bandwidth of nodes in a wireless sensor network are limited so the routing protocol design of a wireless sensor network is very different from the traditional mobile ad hoc network. Important objectives of the routing design of a wireless sensor network are to reduce energy loss and improve the life cycle of the network. Previous tasks of traditional MANET routing protocol design were to provide highquality service in a mobile condition. However, MANET routing protocol cannot be applied to the wireless sensor network due to different design objectives [2].

In recent years, research on WSN routing protocol has become a topic of great interest in the research into wireless sensor networks. Relatively important WSN routing protocols were summarized in this thesis and they are classified into five categories according to the realization feature of protocols: flooding routing protocol, hierarchical routing protocol, routing protocol oriented on data, routing protocol based on position information and routing protocol based on QoS. The most typical routing protocols of these five categories are introduced respectively. The structure of the wireless sensor network and key factors of the routing protocol design are introduced in Section 2; existing WSN routing protocols are classified and the typical routing protocols of each category are introduced in Section 3; routing protocols are analyzed and compared in Section 4; and finally the thesis is summarized.

\section{DESIGN REQUIREMENTS FOR WSN ROUTING PROTOCOL}

\section{A. Structure of wireless sensor network}

Development of a micro electro-mechanical system and low-power and high-integration digital equipment results in low-overhead, low-power and micro-volume sensor node. Such a sensor node is composed of a sensing unit, data processing unit, communication unit and portable power and it can collect data, monitor signal and deliver information.

A wireless sensor network is composed of a large number of low-overhead micro sensor nodes deployed in the monitoring area and it forms an ad hoc network system via wireless communication. It is aimed to sense, collect and process the information of the monitoring object in the area covered by network. The structure of the wireless sensor network is shown in Figure 1. A large number of sensor nodes are distributed in the monitoring area to collect and monitor data of an object and transmit the data processed by collaboration with sink nodes. The sink nodes transmit information to task management nodes via the Internet or communication satellite. Each sensor node in the wireless sensor network combines the functions of node and router of a traditional network [3]. Apart from collection of local information and data processing, it can store, manage and integrate the data forwarded by other nodes and coordinate some special tasks together with other nodes.

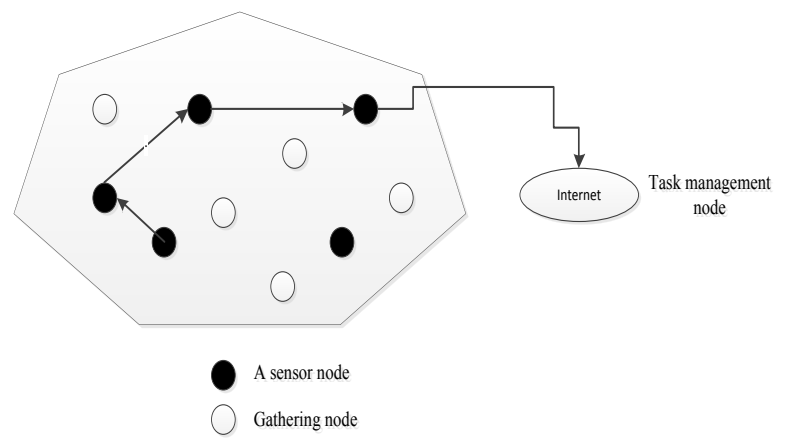

Figure 1. Wireless sensor network structure 
Compared with the traditional wireless MANET network, the wireless sensor network has the following unique features:

(1) Highly restricted resource: as the resource limit of the sensor node is powerful, transmitting power, on-board resource, processing ability, communication bandwidth and storage capacity are all restricted in a low scope.

(2) No global identification: the number of sensor nodes is great and the overhead would be very large if not maintaining global identification. Therefore, being different from traditional routing protocol, global identification is generally not adopted in the sensor network $[4,5]$;

(3) Many-to-one communication: different from pointto-point communication of a traditional network, almost all the applications in the sensor network require multiple source sensor nodes to transmit the data acquired to specific sink nodes [7].

(4) Big data redundancy: multiple source sensor nodes can acquire large amounts of similar data so the amount of data redundancy of the sensor network is large. As wireless sensor network is greatly different from the traditional MANET, and the design objectives and realization methods of their routing protocols are different. Therefore, MANET routing protocol cannot be directly applied in the wireless sensor network.

\section{B. Key factors of WSN routing protocol design}

The major technical challenge of wireless sensor network is to complete sensing, communication and control functions when energy resource, computing power, storage space and communication ability of nodes are highly restricted. Therefore, the main design objective of the routing protocol of the wireless sensor network is to establish an effective energy path, form a reliable data forwarding mechanism and realize the maximum life cycle of network. The structure of the wireless sensor network has the following key factors affecting its routing protocol design.

(1) Network dynamic: most network system structures assume the sensor node is static and the sink node is movable. Whether the monitoring object is moving or static depends on the specific application. For example, in the application for military target tracking, the monitoring objective is dynamic and moving, and in the application for forest fire prevention, the monitoring object is still [8].

(2) Network topology: divided into fixed and ad hoc topology configurations. In a fixed topology, sensor nodes are configured manually and data are transmitted via the preset path; in an ad hoc topology, nodes are scattered randomly in an ad hoc form.

(3) Data sending mode: the data sending mode can be divided into continuous mode, event-driven mode, request-driven mode and mixed mode subject to different application demands. Continuous mode means that the sensor nodes send data periodically subject to preset time intervals; event-driven mode and request-driven mode mean that the sink nodes produce a corresponding event or request to trigger data sending; mixed mode is a combination of the above modes.

(4) Node type: generally all the sensor nodes are isomorphic. If a sensor with a different function is needed in a specific application, there are isomorphic sensor nodes. Recently, some suggest replacing the sensor node with three functions of data forwarding, sensing and collecting with special node with weak resource limitation.

(5) Path selection: there are two selection modes of multi-hop and single hop. Sending energy of broadcast frequency identification is in direct proportion to the square of the distance. As energy consumption of the multi-hop path is less than that of a single-hop path, the multi-hop path is adopted. However, the overhead of topology management and link connection of a multi-hop path is large, so a single-hop path is more effective when the distance between the sensor node and sink node is short.

As WSN is highly correlative with application, the WSN routing protocol has diversity as well and it is difficult to evaluate which protocol is better. Generally, the following indexes are required to evaluate whether the routing protocol design of a wireless sensor network is successful.

(1) Energy effectiveness/life cycle: energy effectiveness is the most important factor in the sensor network design. Reducing energy consumption as much as possible to prolong the life cycle of the network is the primary goal of WSN routing protocol design [9].

(2) Reliability/fault tolerance: sensor node loses are easily due to energy exhaustion or environmental interference, but a partial sensor nodes' lose will not affect the task of the entire network.

(3) Scalability: there may be hundreds or thousands of sensor nodes in some applications and the routing design will collaborate with a large number of nodes.

(4) Delay: delay time of the sensor network refers to the time from observer sending the request to receiving response information. Delay must be reduced as much as possible during the design of the routing protocol.

\section{WSN ROUTING PROTOCOL ANALYSIS}

\section{A. Classification of WSN routing protocol}

Data are transmitted reliably between sensor nodes and sink nodes in the wireless sensor network via the protocol. As the wireless sensor network is highly correlative with the application, a single routing protocol cannot meet different application demands. Subject to the features of different applications, many routing protocols have been studied. These protocols can be generally classified into five categories: flooding routing protocol, hierarchical routing protocol, data-oriented routing protocol, positionbased routing protocol and QoS-based routing protocol.

(1) Flooding routing protocol: this is an old routing protocol. It does not need topology structure to maintain network and routing computation and the node receiving information would directly forward the data package to an adjacent node. For an ad hoc sensor network, flooding routing is a relatively direct method, but it can case message "implosion" and "overlap" easily. Energy limit is not considered here so it has a flaw of a "blind spot of resource".

(2) Hierarchical routing protocol: the basic idea of such a protocol is to cluster sensor nodes and communication within a cluster is completed by cluster head nodes. Cluster head nodes can gather and integrate data to reduce the transmission amount, and finally the cluster head node can send integrated data to the sink node. This mode can meet the scalability of the sensor network and maintain the 
energy consumption of the sensor node to prolong the life cycle of the network.

(3) Data-oriented routing protocol: this protocol names the data in the sensor network in a specific description method. Data transmission is based on data request and depends on data naming. All the data communications are restricted within a local area. Such communication no longer relies on a specific node but relies on the data in the network, so a large number of repeated and redundant data transmitted in in network is reduced, unnecessary overhead is saved and network life cycle is prolonged.

(4) Position-based routing protocol: this forwards requests or data to the needed area by use of position information of a node to narrow the transmission scope of data. In fact, many routing protocols of the sensor network assume the position of a node is given, so nodes can be divided into different domains subject to position information of a node. Data transmission based on domain can narrow transmission range, reduce the communication amount of intermediate nodes and prolong the life cycle of the network.

(5) QoS-based routing protocol: the energy-aware QoS route must guarantee the effective use of band width and efficient energy path in the entire connection time. QoSbased routing protocol applies to real-time applications such as real-time tracking of military targets and emergency event monitoring.

\section{B. Flooding routing protocol}

\section{(1) Flooding protocol}

Flooding is a traditional flooding routing technology. It need not maintain the topology structure of the network to implement routing computation. Node receiving information can forward data packages to all the adjacent nodes in broadcast form. This process will be executed repeatedly until the data package reaches its destination or reaches the preset maximum hop number.

Although the idea of flooding protocol is simple and easy to implement, it has defects of message implosion, massage overlap and blind spots of resource. Message implosion means the same message is sent to the same node via different paths. As shown in Figure 2, node D receives two messages sent from node A. Message overlap means nodes that are placed in the same area monitor the same message and they generate a similar data package to the same adjacent nodes. As shown in Figure 3, node C receives a message about $r$ from nodes $A$ and $B$. Blind spot of resource means a large amount of energy is consumed while the limit of energy use is not considered.

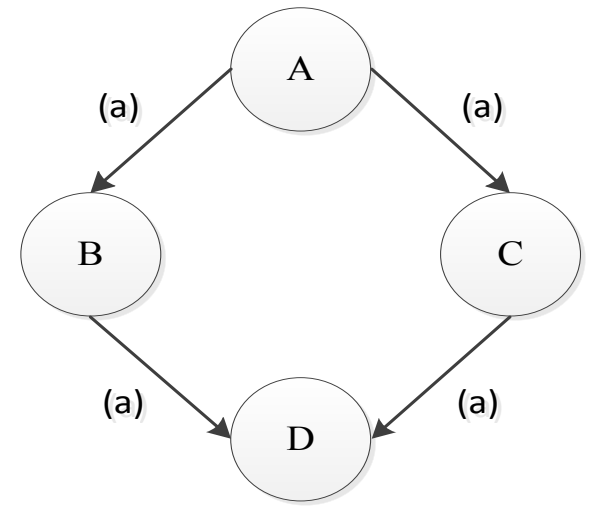

Figure 2. Flooding protocol implosion

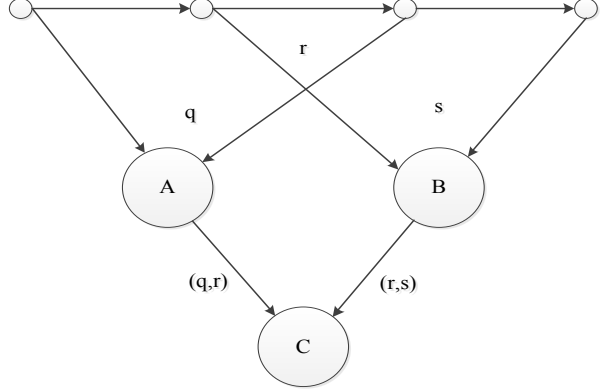

Figure 3. Flooding protocol messages overlap

\section{(2) Gossiping protocol}

Gossiping protocol is the improvement of flooding protocol and nodes that send data do not transmit data in broadcast form, but rather forward data to an adjacent node at random.

Gossiping protocol can avoid message implosion and save energy but it cannot solve the problems of partial message overlap and overuse of resources, and it may increase the transmission delay from terminal to terminal. A simple example about the delay of gossiping protocol is given in Figure 4. Node $\mathrm{F}$ needs the data produced by node $\mathrm{A}$. If node $\mathrm{C}$ selects an adjacent node $\mathrm{D}$ to forward data copy $(\mathrm{X})$ at random in the transmission, finally node $\mathrm{F}$ will receive the data. In this way, node $\mathrm{C}$ not only receives data copy $(\mathrm{X})$ twice but also delays $\mathrm{A}->\mathrm{B}->\mathrm{C}->\mathrm{E}-$ $>$ F into $\mathrm{A}->\mathrm{B}->\mathrm{C}->\mathrm{D}->\mathrm{C}->\mathrm{E}->\mathrm{F}$. As a result, transmission delay is increased.

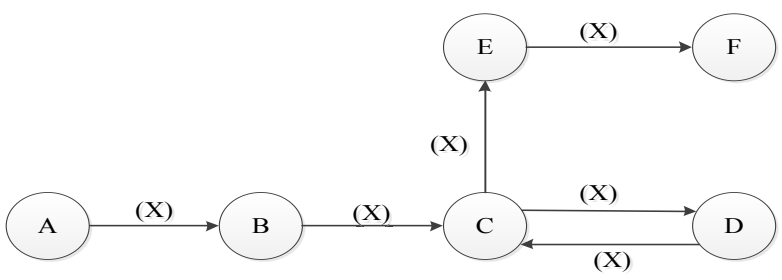

Figure 4. Gossiping agreement delay

\section{Hierarchical routing protocol}

LEACH (Low-Energy Adaptive Clustering Hierarchy) protocol [6] is the first hierarchical routing protocol in the wireless sensor network. Most subsequent hierarchical routing protocols were evolved from $\mathrm{LEACH}$.

The main idea of the protocol is to select a cluster head node at random and evenly share the relay communication service of the wireless sensor network in order to evenly consume the energy of nodes in the sensor network and further prolong the life cycle of the network. LEACH protocol has two stages: cluster preparation stage and data transmission stage. The total number of times of these two stages is called a cycle.

In the cluster preparation stage, a node is selected as a cluster head at random. The cluster head node broadcasts messages to the surroundings, and other nodes select a cluster to join in, subject to the intensity of the broadcasted messages they received, and then inform the corresponding cluster heads. A cluster head communicates with a sink node directly and the cluster members only communicate with the cluster head in their own cluster. The establishment process of a cluster head is to first select a number in the random numbers from node 0 to node, and if the number in the current cycle is smaller than the 
threshold value $T(n)$, the node is the cluster head node. The computation formula of $\mathrm{T}(\mathrm{n})$ is as follows:

$$
T(n)=\left\{\begin{array}{l}
\frac{p}{1-p \times\left(r \bmod \frac{1}{p}\right)}, n \in G \\
0, \quad \text { others }
\end{array}\right.
$$

Where $\mathrm{p}$ is the percentage of expected cluster head nodes in sensor nodes, $r$ is current cycle number and $G$ is the set of nodes that do not become cluster head nodes in the last $1 / \mathrm{p}$ cycle. In the data transmission stage, nodes collect monitoring data constantly and transmit them to the cluster head, and the cluster head integrates data and sends them to the sink node. The cluster head is reselected in the next working cycle.

LEACH protocol selects cluster heads at random to guarantee the high energy consumption overhead of data transmission between cluster head and sink node is evenly shared by all the sensor nodes so that nodes that lose effect due to energy exhaustion are distributed at random. When compared with common multi-hop routing protocol and static cluster algorithm, LEACH can prolong the life cycle of a network by $15 \%$. As LEACH assumes all the nodes can directly communicate with cluster head nodes and sink nodes and adopts continuous data transmission mode and single-hop path selection mode, it does not apply to the application of a large monitoring range and the dynamic cluster brings extra overhead of topology change and a large broadcasting amount.

(2) TEEN protocol

TEEN protocol has the same cluster mode as LEACH protocol, but the cluster head nodes form a hierarchical structure subject to the distance between them and the sink nodes. TEEN protocol has a corresponding reaction to a dramatic change in the sensing attribute. This characteristic is favorable for network operation in an interactive mode in a real-time environment. For each sensing attribute, TEEN protocol defines hard and soft thresholds to determine whether to send the data of this attribute. Data can be sent out only when the following two conditions are met:

(1) The attribute value of current data is larger than the hard threshold.

(2) The difference between the attribute value of current data and the attribute value sent previously is larger than the soft threshold. TEEN protocol forms a cluster via data center so adjacent sensor nodes form a cluster. The process proceeds constantly until the cluster reaches the sink nodes. The hierarchical structure after TEEN protocol generates clusters is shown in Figure 5. When a cluster is formed, the cluster head node will broadcast soft and hard thresholds. As users generally do not need all the data, cyclic data transmission is unnecessary. Therefore, the TEEN protocol saves much more data transmission overhead than the LEACH protocol.

A defect of TEEN protocol is that if an attribute value of data cannot reach the threshold, the node will not send out data and the user cannot receive any data from the network and cannot know whether all the nodes are dead or not.

\section{(3) PEGASIS protocol}

PEGASIS protocol is established on the basis of LEACH protocol. The node in PEGASIS only communicates with the adjacent node that is the closest to it to avoid a large amount of communication overhead caused

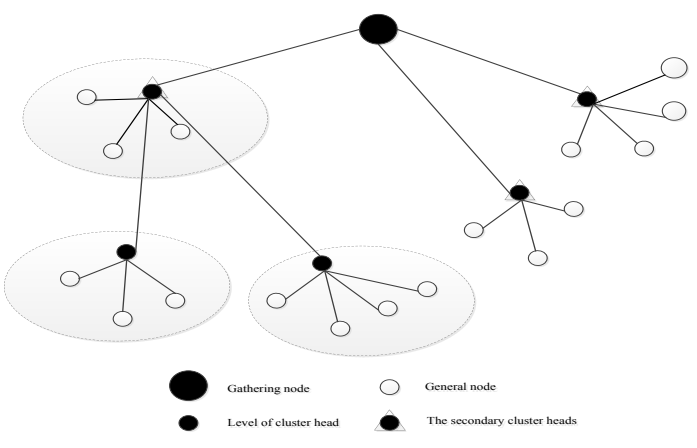

Figure 5. TEEN protocol generates clusters

by frequent selection of a cluster head. All the nodes form one cluster and one node is selected as the cluster head in every cycle. In PEGASIS protocol, a chain is formed by a greedy algorithm, which means a node sends out progressive energy decrease signal to find the adjacent node closest to itself to establish a chain that contains all the nodes in the network as shown in Figure 6 (a). Then the cluster head is selected dynamically. Assuming $\mathrm{N}$ nodes in the network are numbered by $1-\mathrm{N}$ natural numbers and the cluster head selected in the $\mathrm{j}_{\text {th }}$ cycle is the $\mathrm{i}_{\text {th }}$ node, then $\mathrm{i}=\mathrm{j} \bmod _{\mathrm{N}}$ (value is $\mathrm{N}$ when $\mathrm{i}=0$ ) is satisfied and the cluster head communicates with the sink nodes via a single hop.

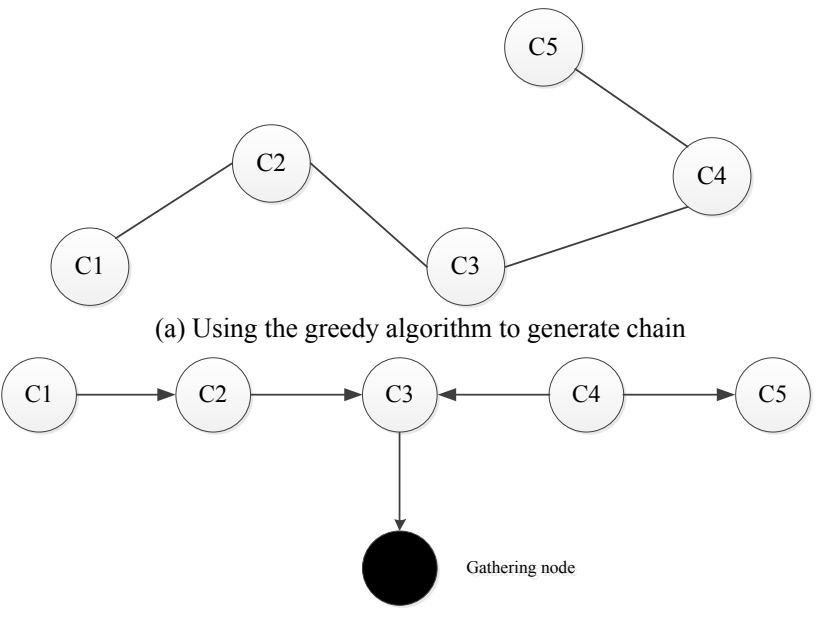

(b) The path of the data transmission to the gathering node

Figure 6. PEGASIS protocol

When a cluster head is selected, nodes at both ends of the chain send data to the next nodes towards the middle of the chain and nodes that receive data integrate their data with received data and send the integrated date to the next nodes. Finally all the data are transmitted to the cluster head node. The cluster head node then integrates two groups of data and its own data and forms a data package and sends it to the sink node as shown in Figure 6 (b) (c3 is cluster head). If the node that is far from the receptor is the cluster head, the communication overhead between the node and sink node is too large, which will result in premature death of the node. So, PE-GASIS sets a distance threshold value. If the distance between the node and the sink node exceeds the threshold value, the node will not be the cluster head. When the data from the two ends are all transmitted, a new cycle of selection and transmission will begin. 
PEGASIS protocol avoids large amounts of communication if LEACH protocol causes frequent selection of cluster heads, and it provides an effective chain-type data aggregation, which significantly reduces the number of times and amount of communication data transmission. Nodes always communicate with the closest node and form multi-hop communication mode so energy is effectively reduced and the lifetime of the network is increased dramatically. Simulation result shows that when compared with LEACH protocol, the life cycle of the sensor network of PEGASIS protocol can be prolonged one to three times for different applications and network topology structures. The defects of PEGASIS protocol are that a single cluster method makes the cluster head become the key point and its failure would result in routing failure. Furthermore, all the sensor nodes must have the ability to communicate with the sink node, and if the chain is too long, data transmission delay will be increased so it does not apply to real-time application. Finally, the chaining algorithm requires nodes to know positions of other nodes so the overhead is very large.

\section{Data-centered routing protocol}

\section{(1) SPIN protocol}

SPIN protocol is the earliest data-centered routing protocol which solves problems of "implosion" and "overlap" in arithmetic through a negotiation mechanism. Nodes of SPIN protocol use three kinds of information to communicate: ADV, REQ and DATA. After nodes generate or receive the data, to avoid blind spread, ADV information which contains metadata is used to notify adjacent nodes, while adjacent nodes which need data make a request using REQ information, and data is sent to the request node through DATA information.

Figure 7 shows the route setup and data transmission of SPIN protocol. In Figure 7 (a), node A notifies node B of data description which it possesses; node B makes a request to node $\mathrm{A}$ in Figure 7 (b); node A transfers data to node B in Figure 7 (c); Figure 7 (d) shows that node B has received information, and sends ADV information to its adjacent node; Figure 7 (e) shows that node which needs this data sends REQ information to node B; Figure 7 (f) shows that node $\mathrm{B}$ transfers data to the request node.

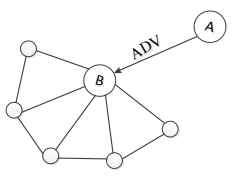

(a)

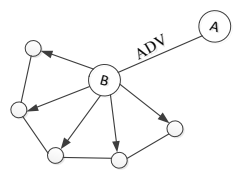

(d)

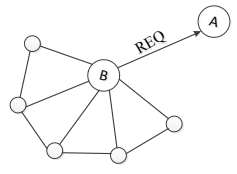

(b)

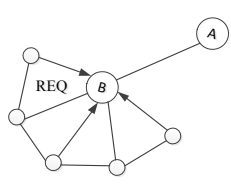

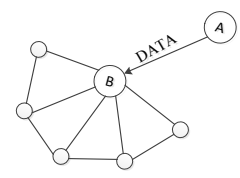

(c)

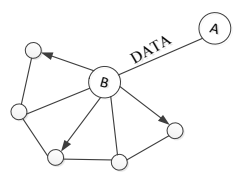

(f)
Figure 7. SPIN protocol routing establishment and data transmission

The advantages of SPIN protocol are that small ADV information reduces the problem of implosion; the overlapping of information is resolved through data naming; nodes decide whether to make an ADV notice according to their own resources and application information, thus avoiding the problem of blind utilization of resources. Compared with flooding and gossiping, SPIN protocol saves energy. The disadvantages of SPIN protocol are that when all adjacent nodes of nodes that generate or receive data do not need this data, it will make data unable to further transmit, and relatively farther nodes may be unable to receive the needed data; when a sink node needs all data, its adjacent nodes easily consume energy; SPIN protocol reduces the data implosion, but ADV implosion still exists.

(2) Directed Diffusion Protocol [10] was a milestone in the development process of data-centered routing protocol, and other data-centered routing protocols are all improvements based on this protocol or are proposed with similar key ideas. The main idea of Directed Diffusion Arithmetic is to use one set of properties to name data on a network and communicate based on this data. Directed Diffusion adopts a data transfer mode which is driven by request. A new directed diffusion process will start when a sink node sends a request command to a certain event, and the process includes the three stages of requests for diffusion, initial gradient establishment and data transmission, as shown in Figure 8.

In the stage of data transmission, the sink node will send a piece of strengthening selection information to an adjacent node which receives the new data first. The adjacent node which receives the strengthening selection also sends a strengthening selection to its adjacent node which firstly receives the new data to spread this request information which bears a larger gradient value, and finally a path with the largest gradient value will be formed. Target data can transmit data by following this strengthen path at a higher data transmission rate, and other paths formed by nodes of lower data transmission rates can be used as alternative paths in order to increase network reliability.

\section{E. Routing protocol based on QoS}

SAR Protocol is the first routing protocol to ensure QoS in wireless sensor networks. In order to improve the energy efficiency and fault-tolerant capability, it provides a multipath routing with table driven. In the protocol, all single-hop nodes adjacent to a sink node create spanning trees by taking themselves as roots. In the process of creating spanning trees, QoS parameters of energy resources, priority of the data packet, and the delay and packet loss probability of nodes are all considered. After the creation of spanning tree is completed, one sensor node may belong to multiple trees, thus reversely establishing multiple paths to sink nodes with different QoS parameters. When transmitting data, a node will select one or several paths to transmit according to energy resources and QoS. Any local error will automatically trigger local path reconstruction in order to realize error recovery by the enforcement of consistency of routing tables of each node on each path. SAR Protocol maintains these multiple paths from sensor nodes to sink nodes. This not only provides QoS guarantee, but also has a fault-tolerant capability, and is easy to recover. But the disadvantage is that a large number of redundant routing information in nodes wastes storage resources, and routing information maintenance, the update of QoS parameters of nodes and energy consumption information all require a larger overhead.

\section{COMPARISON OF WSN ROUTING PROTOCOL CHARACTERISTICS}

This section compares the performance and characteristics of routing protocols of wireless sensor networks from the aspects of life cycle, extendibility, path selection, en- 
PAPER

ANALYSIS AND COMPARISON OF ROUTING PROTOCOL OF WIRELESS SENSOR NETWORK

TABLE I. THE WIRELESS SENSOR ROUTING PROTOCOL

\begin{tabular}{|c|c|c|c|c|c|c|c|c|c|c|}
\hline Algorithm & $\begin{array}{c}\text { The life } \\
\text { cycle }\end{array}$ & $\begin{array}{c}\text { Expand } \\
\text { sex }\end{array}$ & \begin{tabular}{|c|}
$\begin{array}{c}\text { Path selec- } \\
\text { tion }\end{array}$ \\
\end{tabular} & $\begin{array}{c}\text { Energy } \\
\text { awareness }\end{array}$ & $\begin{array}{c}\text { Data ag- } \\
\text { gregation }\end{array}$ & $\begin{array}{c}\text { Location } \\
\text { information }\end{array}$ & $\begin{array}{l}\text { Stored in- } \\
\text { formation }\end{array}$ & Mobile nodes & $\begin{array}{l}\text { Real } \\
\text { time }\end{array}$ & Reliability \\
\hline Flooding & short & bad & Multi-hop & None & None & No need & None & $\begin{array}{l}\text { A sensor node } \\
\text { Gathering node }\end{array}$ & bad & better \\
\hline Gossiping & longer & bad & Multi-hop & None & None & No need & None & $\begin{array}{l}\text { A sensor node } \\
\text { Gathering node }\end{array}$ & bad & better \\
\hline LEACH & longer & bad & Single hop & have & have & No need & have & None & bad & better \\
\hline TEEN & longest & good & Multi-hop & have & have & No need & have & None & good & bad \\
\hline PEGASIS & longest & bad & Multi-hop & have & have & No need & have & None & bad & bad \\
\hline SPIN & longest & bad & Multi-hop & None & have & No need & None & $\begin{array}{l}\text { A sensor node } \\
\text { Gathering node } \\
\end{array}$ & bad & bad \\
\hline DD & longest & good & Multi-hop & have & have & No need & have & $\begin{array}{l}\text { A sensor node } \\
\text { Gathering node }\end{array}$ & bad & good \\
\hline Rumor & longest & good & Multi-hop & have & have & No need & have & none & bad & better \\
\hline GPSR & longer & good & Multi-hop & none & none & need & none & $\begin{array}{l}\text { A sensor node } \\
\text { Gathering node }\end{array}$ & bad & better \\
\hline GEAR & longest & good & Multi-hop & have & none & Need & none & $\begin{array}{l}\text { A sensor node } \\
\text { Gathering node }\end{array}$ & bad & better \\
\hline SAR & longer & $\mathrm{Bad}$ & Multi-hop & have & none & No need & Have & None & Good & Good \\
\hline SPEED & longest & bad & Multi-hop & have & none & Need & have & none & good & good \\
\hline
\end{tabular}

ergy awareness, data aggregation, location information, information storage, mobile nodes, real-time and reliability (fault-tolerant ability). Table 1 shows the results of comparison between various kinds of routing protocols as mentioned above. Since the design of routing protocol of wireless sensor networks is closely related to application, in practical application, at the time of selecting the routing protocol, the specific application and characteristics of all routing protocols will be considered comprehensively.

\section{CONCLUSION}

Compared with the traditional wire network and wireless MANET network, resources are highly restricted and nodes are easy to lose efficacy in wireless sensor networks, thus the design of its routing protocol faces new challenges. This paper summarizes and analyzes the research achievements of routing protocols of wireless sensor networks in recent years, and divides them into flooding routing protocol, hierarchical routing protocol, datacentered routing protocol and routing protocols based on location information and QOS. The paper makes corresponding algorithm analysis on each kind, and finally compares and summarizes the characteristics of these protocols. With the continuous development of the application, the routing protocols of wireless sensor networks still have many problems that need further research, and the following are the main points:

(1) Effectiveness of energy: In wireless sensor networks, frequent data communication expends a large amount of energy; reduces data traffic, restrains transmitting unnecessary data on nodes, and improves energy efficiency, which are priorities in the design of WSN routing protocol.

(2) Reliability: Nodes of wireless sensor networks easily lose efficacy. How to calculate routing through network information that is easy to get from nodes, make sure that routing is easy to recover when breaking down, and how to highly reliably transmit data are key matters to be solved in the design of routing protocol.

(3) Real-time: In the near future, real-time transmission of image and video will become a very common applica- tion requirement in wireless sensor networks. The research and design of routing protocol that has a high transmission quality, small transmission delay, and meets real-time demands have a broad application prospect.

(4) Security: The inherent characteristics of wireless sensor networks make its routing protocol susceptible to security threats. Application fields of high a security level, such as military applications, present new requirements for the security of routing protocols. Research on such aspects is still in initial stages at present.
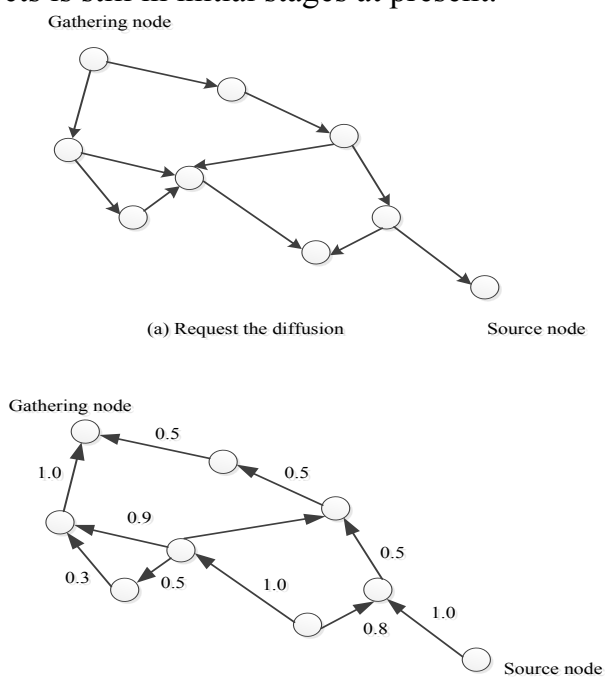

(b) Gradient field to establish

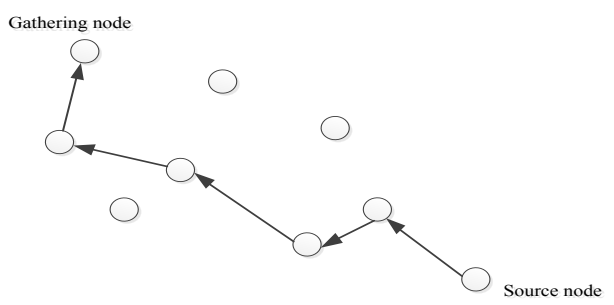

(c) The data transfer

Figure 8. Three stages of Directed Diffusion Protocol 


\section{REFERENCES}

[1] A. Rubeaai, F. S. Sarab, K. Brajendra, "Region based three dimensional real-time routing protocol for wireless sensor networks," Proceedings of IEEE Sensors, pp. 233-236.

[2] H. A. Tom, H. Falah, "Location aware sensor routing protocol for mobile wireless sensor networks," IET Wireless Sensor Systems, vol. 2, pp. 49-57, April 2016.

[3] W. Runze; S. Xiaoping, "An improved ACO-based security routing protocol for wireless sensor networks," Proceedings-2013 International Conference on Computer Sciences and Applications, CSA 2013, pp. 90-93.

[4] M. Kumarasamy, Vallipuram, "An energy aware heuristic-based routing protocol in Wireless Sensor Networks," 2014 17th International Conference on Computer and Information Technology, ICCIT 2014, pp. 53-58.

[5] D. R. Gawade, S.L. Nalbalwar, "A centralized energy efficient distance based routing protocol for wireless sensor networks," Journal of Sensors, vol.21, pp.212-216, April 2016. http://dx.doi.org/10.1155/2016/8313986

[6] Z. Xuedan, D. Yuhan; L. Xiaokang, "Adaptive energy-harvesting aware clustering routing protocol for Wireless Sensor Networks," 2012 7th International ICST Conference on Communications and Networking in China, CHINACOM 2012 - Proceedings, pp. 742747.

[7] P. Kamalakkannan, "Enhanced cluster based routing protocol for mobile nodes in wireless sensor network," Proceedings of the 2013 International Conference on Pattern Recognition, Informatics and Mobile Engineering, PRIME 2013, pp. 187-193.

[8] H. Pulagam, "Multiple aggregator multiple chain routing protocol for heterogeneous wireless sensor networks," 2013 International Conference on Signal Processing and Communication, ICSC 2013, pp. 127-131.
[9] W. Yineng, "Distributed energy aware routing protocol for largescale wireless sensor network," 12th Annual Mediterranean Ad Hoc Networking Workshop, MED-HOC-NET 2013, pp. 111-115.

[10] B. Doina, "An evolutionary framework for routing protocol analysis in wireless sensor networks," Lecture Notes in Computer Science, vol. 7835, pp. 1-11, April 2013. http://dx.doi.org/10.1007/ 978-3-642-37192-9 1

\section{AUTHORS}

Lei An is an associate professor with master degree. He works in department of mathematics and computer, BaoDing University. His research direction is computer network (e-mail: hda19936@126.com).

Aihua Li is a lecture with master degree. She works in department of mathematics and computer, BaoDing University. Her research direction is computer Network (email: liaihua0130@163.com).

Ling Pang is a lecture with master degree. She works in department of information management and engineering, HeBei finance University. Her research directions are computer measurement and control technology (e-mail: 364627233@qq.com).

Guangdong He (corresponding author) is an associate professor with master degree. He works in department of mathematics and computer, BaoDing University. His research direction is data mining (e-mail: 59060777@qq.com).

Submitted 03 September 2016. Published as resubmitted by the authors 05 October 2016. 